\author{
С.А. Ганджа ${ }^{1}$, Б.И. Косимов ${ }^{1}$, Д.С. Аминов ${ }^{1}$, Р.Р. Ниматов ${ }^{2}$ \\ ${ }^{1} Ю$ жо-Уральский государственный университет, Челябинск, Россия \\ ${ }^{2}$ Комсомольский-на-Амуре государственный университет, \\ Комсомольский-на-Амуре, Россия
СРАВНИТЕЛЬНЫЙ АНАЛИЗ ЭЛЕКТРОДВИГАТЕЛЕЙ ПРИВОДА ПИЛЬГЕРСТАНА ДЛЯ ТЕХНОЛОГИИ ИЗГОТОВЛЕНИЯ БЕСШОВНЫХ ТРУБ. ВЫБОР ОПТИМАЛЬНОЙ КОНСТРУКЦИИ

\begin{abstract}
Одним из наиболее перспективных способов изготовления бесшовных труб являются так называемые пилигримовые станы. Это объясняется тем, что переход на разные диаметры труб можно осуществить в минимальные сроки. На данный момент концепция этого способа производства морально не устарела, но существующие станы, построенные несколько десятилетий назад, физически изношены и требуют замены. Данная статья посвящена этой актуальной проблеме. Заказчиком работы является Челябинский трубопрокатный завод, на котором пилигримовый стан работает с 1928 г. По требованию руководства предприятия необходимо заменить существующий коллекторный двигатель на современный высоконадежный привод, при этом повысить энергетические показатели. Требуется рассмотреть варианты разделения существующего привода, работающего одновременно на два цеха, на два раздельных привода, обслуживающего каждый цех. В статье проведен анализ различных вариантов электроприводов на базе асинхронных и синхронных двигателей. Для всех вариантов была использована программа точного расчета электрических машин на основе метода конечных элементов ANSYS Electronics Desktop. Проведен сравнительный анализ выбранных вариантов. В качестве показателей наилучшего варианта были выбраны минимальный расход активных материалов и энергетические показатели. Для замены существующего морально и физически устаревшего коллекторного электродвигателя постоянного тока предложен привод на базе вентильного электродвигателя с когтеобразными полюсами с постоянными магнитами, который имеет более высокую надежность за счет бесконтактного токоподвода, минимальный расход меди, лучшие энергетические показатели за счет исключения потерь на возбуждение.

Ключевые слова: пильгерстан, асинхронный электродвигатель, синхронный электродвигатель, тихоходный электродвигатель, постоянные магниты, электромагнитное возбуждение, когтеобразные полюса.
\end{abstract}


S.A. Gandzha', B.I. Kosimov ${ }^{1}$, D.S. Aminov ${ }^{1}$, R.R. Nimatov ${ }^{2}$

${ }^{1}$ South Ural State University, Chelyabinsk, Russian Federation

${ }^{2}$ Komsomolsk-on-Amur State University, Komsomolsk-on-Amur, Russian Federation

\section{COMPARATIVE ANALYSIS OF ELECTRIC MOTORS OF PILGER MILL DRIVE FOR TECHNOLOGY OF MANUFACTURING SEAMLESS PIPES. CHOICE OPTIMAL DESIGN}

One of the most promising methods for the manufacture of seamless pipes are the so-called pilgrim mills. This is because the transition to different pipe diameters can be accomplished in the shortest possible time. At the moment, the concept of this mode of production is not morally obsolete, but the existing mills, built several decades ago, are physically worn out and require replacement. This article focuses on this pressing issue. The customer is the Chelyabinsk Pipe Rolling Plant, where the pilgrim mill has been operating since 1928. At the request of the company's management, it is necessary to replace the existing collector engine with a modern, highly reliable drive, while increasing energy performance. It is required to consider options for dividing an existing drive operating simultaneously into two workshops into two separate drives serving each workshop. The article analyzes various variants of electric drives based on asynchronous and synchronous motors. For all the options, the program of accurate calculation of electric machines based on the ANSYS Electronics Desktop finite element method was used. Comparative analysis of the selected variants is performed. The minimum consumption of active materials and energy indicators were chosen as indicators of the best option. To replace the existing morally and physically obsolete collector DC motor, a drive based on a valve motor with a claw-shaped poles with permanent magnets has been proposed, which has higher reliability due to a non-contact current lead, minimum copper consumption, better energy performance, due to exclusion of excitation losses.

Keywords: asynchronous motor, synchronous motor, low-speed motor, permanent magnets, electromagnetic excitation, claw-pole machine.

Введение. Совершенствование технологии трубопрокатного производства является очень актуальной задачей для Российской Федерации, поскольку нефтегазодобывающая отрасль является бюджетообразующей для страны. Среди разнообразного ассортимента труб наиболее надежными являются бесшовные трубы. Бесшовные трубы изготавливаются различными способами. Пилигримовые станы (пильгерстаны) представляют собой двухвалковые трубопрокатные станы для периодической прокатки труб в валках переменного калибра, которые являются одним из способов производства таких труб. Пилигримовый метод является одним из самых экономичных и универсальных способов производства бесшовных труб, так как переход на трубы другого размера на пильгерстане занимает гораздо меньше времени, чем, например, на станах, изготавливающих трубы со сварным швом. Поэтому, несмотря на востребованность новых прокатных технологий, производство труб на пилигримских агрегатах продолжает оставаться од- 
ним из самых перспективных в мире для производства горячекатаных труб широкого спектра типоразмеров и марок сталей. В настоящее время эта технология уникальна. Только три предприятия в России имеют действующие производства: Северский трубный завод (город Полевской), Первоуральский новотрубный завод (Первоуральск), Челябинский трубопрокатный завод (Челябинск).

На Челябинском трубопрокатном заводе (ЧТПЗ) пильгерстан работает с 1928 г. В качестве приводного двигателя используется коллекторный двигатель постоянного тока GM 900/100 фирмы Simens co следующими основными параметрами: номинальная мощность 2,75 МВт, номинальное напряжение питания 6 кВ постоянного тока, номинальная скорость вращения 35 об/мин. Электродвигатель вращает маховик диаметром 9 м с большой инерционной массой 120 т. Привод параллельно работает на два цеха по производству бесшовных труб. Электродвигатель и маховик представлены на рис. 1.

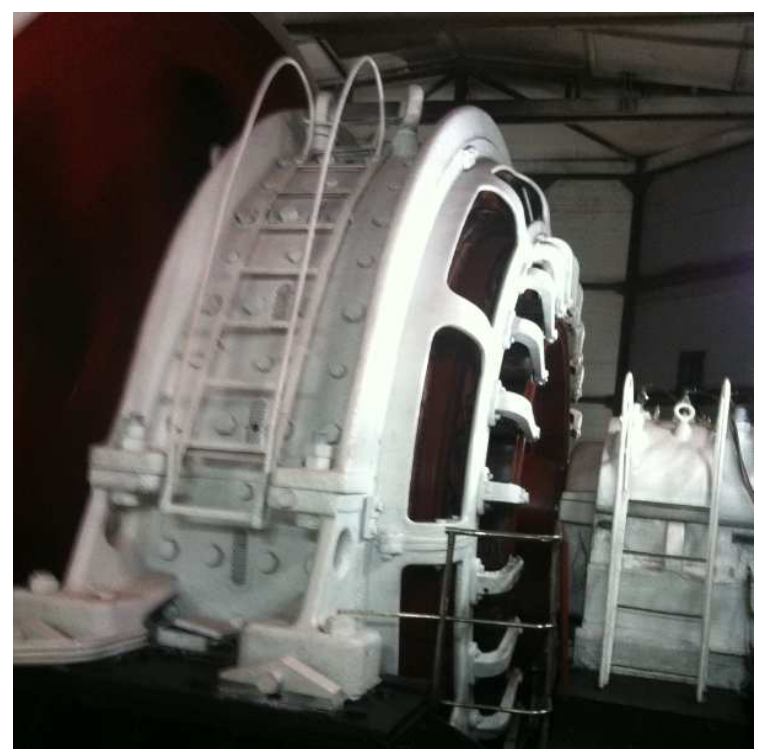

Рис. 1. Электродвигатель, маховик и подшипник пильгерстана ЧТПЗ

Длительная эксплуатация привода привела к его физическому и моральному износу. На валу образовались микротрещины, которые могут привести к его разрушению при ударных нагрузках. Коллектор, обмотка возбуждения, обмотка якоря, подшипниковые узлы требуют периодического ремонта и постоянного дорогостоящего обслуживания. 
Предприятие планирует заменить существующий электродвигатель на более современный, надежный и экономичный вариант бесконтактного электродвигателя.

Данная статья посвящена этой актуальной теме - выбору оптимального варианта электродвигателя для привода прокатного стана ЧТПЗ. Анализ силовой электроники и системы управления приводом является отдельным исследованием, которое будет представлено в последующих публикациях.

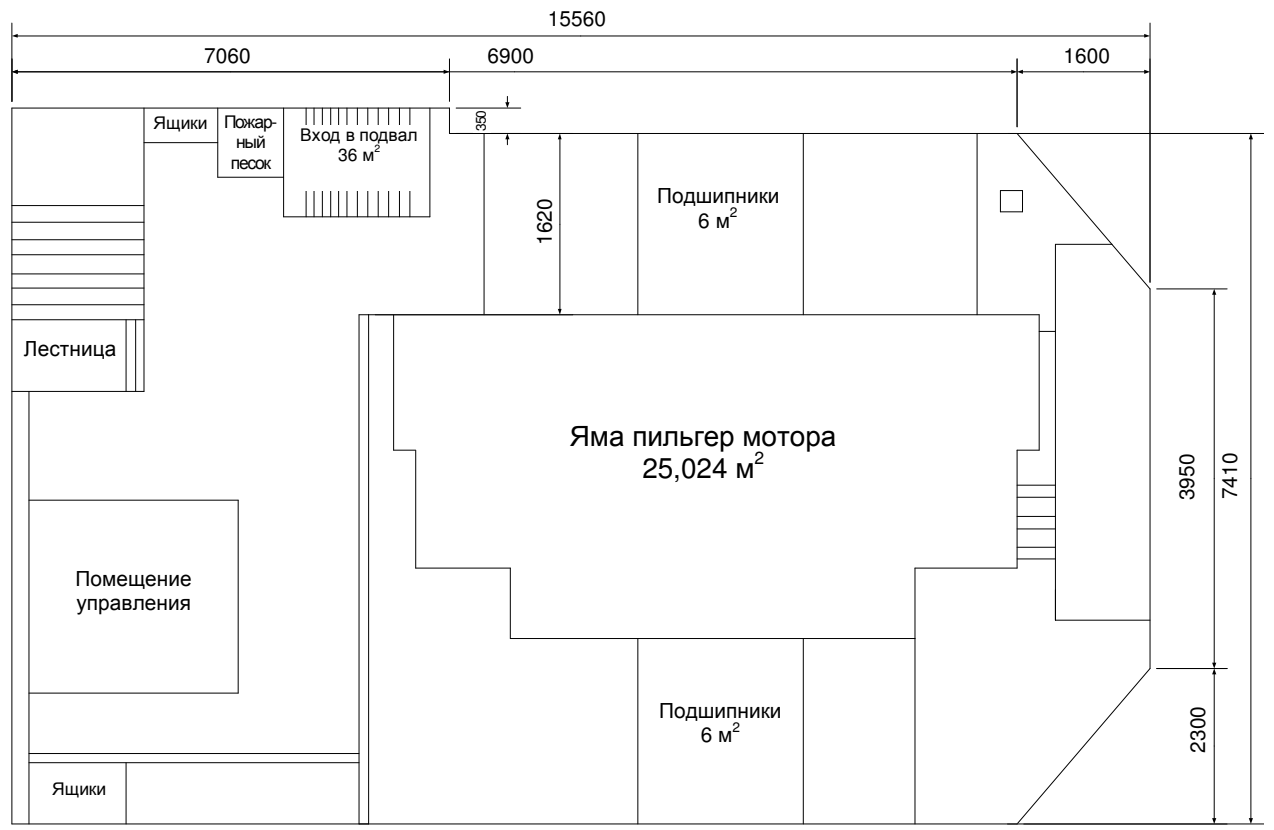

Рис. 2. План размещения существующего привода

\section{1. Требования, предъявляемые к модернизированному при-} воду. При модернизации основные узлы и агрегаты привода по условию предприятия должны остаться без изменений (двухвалковая клеть с калибром переменного сечения и подающий механизм). Планируется замена только электродвигателя и, возможно, маховика.

К новому приводу со стороны руководства предприятия были сформулированы следующие основные требования:

- привод должен соответствовать размерам помещения, в котором находится существующее устройство. Компоновка привода показана на рис. 2;

- для повышения надежности и снижения эксплуатационных расходов привод должен быть бесконтактным; 
- привод должен иметь возможность регулировки частоты вращения в диапазоне 30-45 об/мин для прокатки труб различного диаметра из различных сталей;

- необходимо проработать вариант разделения существующего привода, обслуживающего параллельно два цеха, на два независимых привода меньшей мощности. При этом упростятся регламентные работы по обслуживанию и ремонту;

- необходимо улучшить КПД привода для снижения эксплуатационных потерь;

- необходимо предусмотреть минимальные сроки демонтажа старого привода и его замены новым приводом. Недопустимо прерывание существующей производственной линии на длительный период времени;

- двигатель и система управления должны иметь минимально возможную стоимость.

2. Постановка задачи. Жесткие и противоречивые требования заказчика заставляют учитывать максимально возможное количество конструкций.

Класс реактивных электродвигателей [1-6] был исключен из рассмотрения на начальном этапе, поскольку эти электрические машины в одинаковых габаритах и при одинаковых условиях эксплуатации уступают машинам с активным возбуждением по КПД и другим энергетическим показателям, что теоретически подтверждено и доказано [7-9].

Исходя из принципа действия, предлагается рассмотреть следующие группы вариантов.

Первая группа вариантов связана с использованием высокоскоростного электродвигателя и редуктора.

Вторая группа вариантов связана с использованием прямого безредукторного привода и тихоходного электродвигателя. В качестве бесконтактного электродвигателя возможны варианты асинхронного, синхронного или вентильного электродвигателя [10-19, 21-24]. Ниже приведен список проанализированных вариантов:

1. Высокоскоростной асинхронный электродвигатель с редуктором 2750 кВт.

2. Замена существующего электродвигателя постоянного тока на следующие варианты:

- асинхронный электродвигатель 2750 кВт;

- синхронный электродвигатель 2750 кВт. 
3. Встраивание нового электродвигателя в маховик:

- асинхронный радиальный электродвигатель 2750 кВт;

- асинхронный торцевой электродвигатель 2750 кВт.

4. Исключение маховика и замена его электродвигателем большого диаметра с эквивалентной инерционной массой:

- асинхронный электродвигатель 1375 кВт;

- синхронный электродвигатель с когтеобразными полюсами с постоянными магнитами 1375 кВт.

Необходимо проанализировать выбранные варианты с точки зрения их энергоэффективности при наименьших затратах на замену существующего привода. Рассмотрим все эти варианты последовательно.

3. Вариант зубчатой передачи с высокоскоростным асинхронным двигателем 2750 кВт. Для этого варианта используются высокоскоростной малогабаритный электродвигатель и редуктор с передаточным числом 1:100. В качестве зубчатого элемента можно использовать существующий маховик, если сделать зубья на его наружном диаметре (или использовать накладки с зубьями).

Преимуществом этого варианта является возможность поставки готового серийного высокоскоростного электродвигателя. При этом не требуется затрат на его разработку и изготовление.

Недостатками данного варианта являются:

- разработка нового редуктора, который, как показывает анализ имеющихся информационных источников, не может быть приобретен как готовый компонент;

- необходимость смазки и обслуживания;

- снижение долговечности редуктора из-за больших нагрузок и больших моментов;

- недостаток места в существующем цехе для второго привода.

Для большей наглядности трехмерная модель варианта представлена на рис. 3.

Следует отметить, что этот вариант был рассмотрен техническими специалистами предприятия. Серийный асинхронный электродвигатель был куплен, но позже этот вариант был отклонен из-за перечисленных выше недостатков.

4. Варианты прямого безредукторного привода. Для вариантов с прямым приводом необходима разработка нового электродвигателя, поскольку скорость и момент привода являются уникальными и не существуют для этих параметров серийных электродвигателей. Все по- 
следующие варианты электродвигателей были рассчитаны на кафедре теоретических основ электротехники ЮУрГУ с использованием лицензионной программы Ansys Electronics Desktop [20].

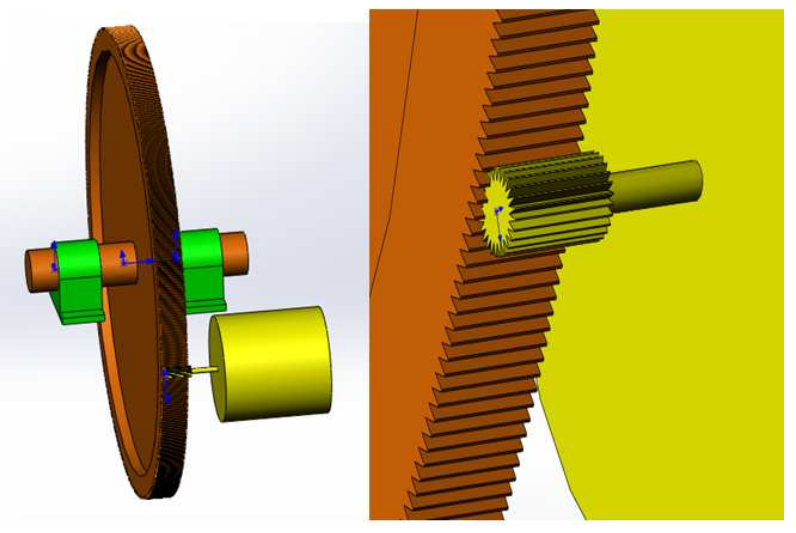

Рис. 3. Трехмерная модель редукторного привода

\section{Замена существующего электродвигателя на асинхронный} электродвигатель 2750 кВТ, 600 В, 35 об/мин. Преимуществом этого варианта является использование существующего маховика и стояковых подшипников, при этом заменяется только сам электродвигатель. Это в значительной степени экономит средства на модернизацию.

Трехфазные асинхронные электродвигатели с короткозамкнутым ротором хорошо изучены и широко применяются в современных системах электропривода [7-9]. При наличии современной системы управления асинхронные электродвигатели не уступают по регулирующим свойствам как двигателям постоянного тока, так и электродвигателям других типов. Однако такие машины имеют худшие удельные массоэнергетические показатели, чем синхронные машины с постоянными магнитами и вентильные двигатели [7-9, 12-14].

На рис. 4 показана трехмерная модель активных частей асинхронного двигателя и результаты расчета его основных параметров.

Двигатель имеет размеры существующего двигателя постоянного тока (около 6 м в наружном диаметре).

Покажем преимущества данного варианта:

- электродвигатель вписывается в существующие габариты электродвигателя постоянного тока;

- электродвигатель имеет бесконтактный токоподвод, т.е. обладает высокой надежностью, как все асинхронные электродвигатели. 

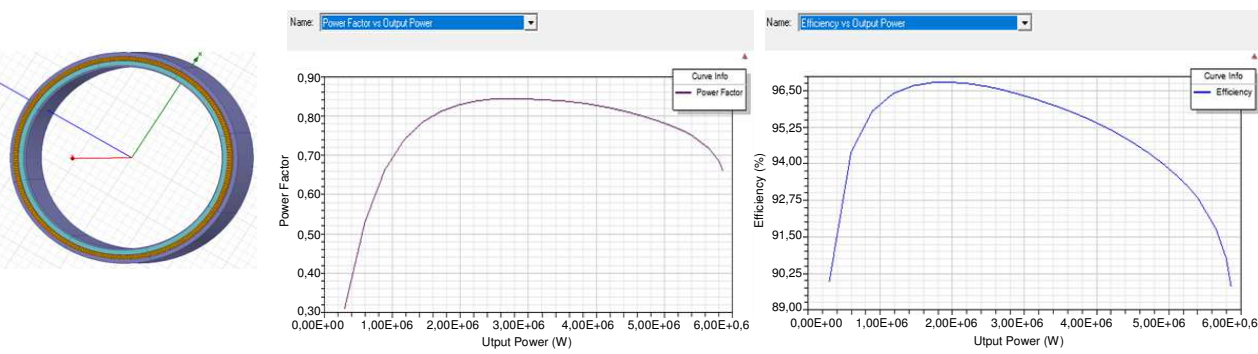

Рис. 4. Трехмерная модель активных частей асинхронного электродвигателя, графики коэффициента мощности и КПД

К недостаткам этого варианта можно отнести следующее: электродвигатель обладает не очень высокими энергетическими показателями (КПД и коэффициентом мощности). При высоких номинальных мощностях и длительной непрерывной работе это приведет к увеличению расходов на эксплуатацию привода.

Замена существующего электродвигателя на синхронный электродвигатель с электромагнитным возбуждением 2750 кВт, 600 В, 35 об/мин. Как и в предыдущем рассмотренном варианте, замене подлежит только электродвигатель. Маховик и подшипники остаются без изменений. Этот вариант был проанализирован, потому что из теории электрических машин хорошо известно, что синхронный двигатель, при прочих равных условиях, имеет лучшие удельные показатели по сравнению с асинхронным двигателем.

Он имеет высокую перегрузочную способность и жесткую механическую характеристику, которая слабо зависит от напряжения питания и нагрузки [12-13]. На рис. 5 показана конструкция электродвигателя и основные ее фрагменты.
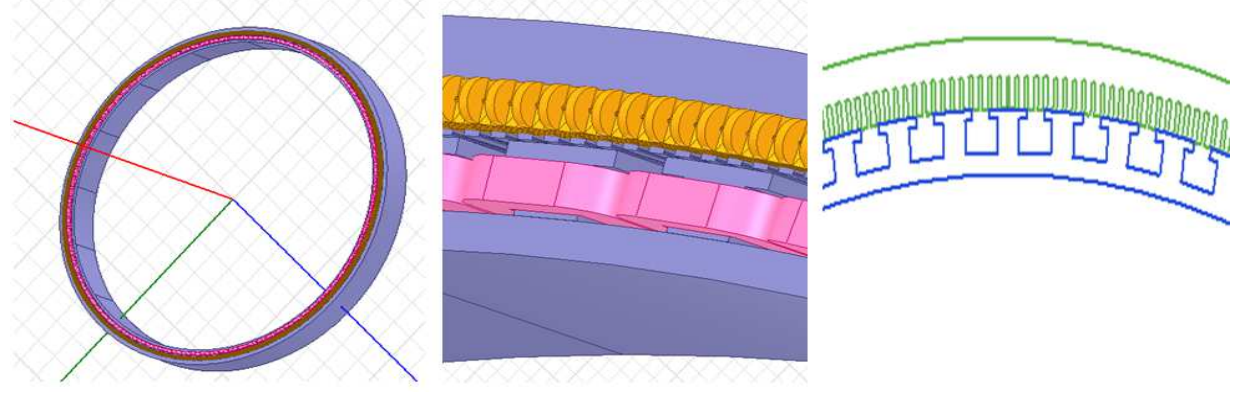

Рис. 5. Конструкция синхронного электродвигателя с электромагнитным возбуждением 
На рис. 6 представлены зависимости выходной мощности и КПД электродвигателя.
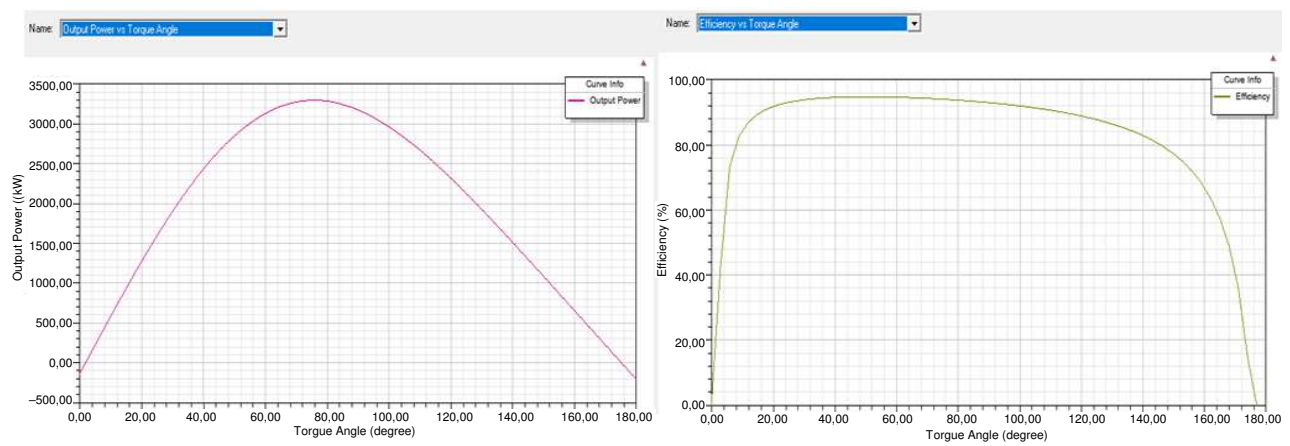

Рис. 6. Зависимости мощности и КПД от угла нагрузки

Отметим достоинства этого варианта:

- электродвигатель вписывается в габариты существующего электродвигателя постоянного тока;

- электродвигатель имеет улучшенные энергетические показатели: КПД и коэффициент мощности.

К недостаткам варианта следует отнести следующее:

- электродвигатель имеет контактный токоподвод для возбуждения, что снижает надежность и требует дополнительного обслуживания;

- электродвигатель имеет габариты, не позволяющие разместить такой же второй независимый привод.

4. Варианты электродвигателей, которые используют существующий маховик в качестве ротора электродвигателя. Известно, что мощность электродвигателя зависит от диаметра в квадрате [7]. Большие размеры маховика (9 м) позволяют рассмотреть возможность использования его в качестве ротора электродвигателя. Этот вариант привлекателен тем, что при модернизации можно не останавливать производство, проводя монтаж вокруг маховика и эксплуатируя старый электродвигатель. При этом снижаются технические риски в случае, если новый двигатель не подтвердит свои энергетические характеристики. В этой группе вариантов мы рассмотрим радиальные и торцевые электродвигатели. Синхронные двигатели были исключены из рассмотрения из-за сложности монтажа ротора с постоянными магнитами.

Радиальный асинхронный электродвигатель 2750 кВт, 600 В, 35 об/мин, встраиваемый в существующий маховик. Максимальное радиальное биение маховика, измеренное специалистами завода, 
составило 10 мм. Это обстоятельство нужно было учитывать при выборе воздушного зазора. На рис. 7 показана трехмерная модель варианта. Результаты расчета электродвигателя представлены на рис. 8.
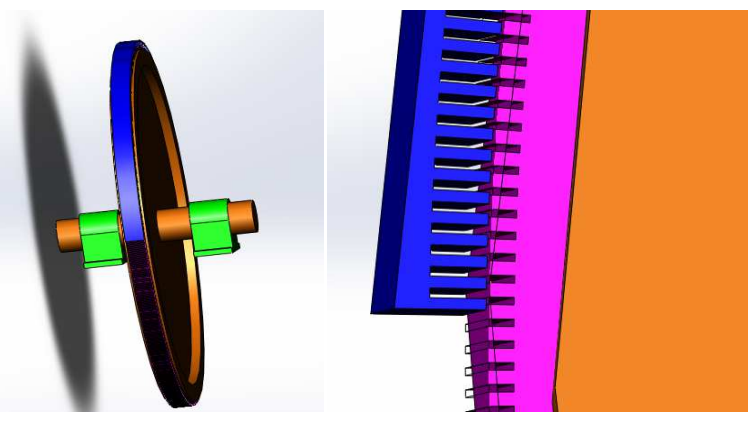

Рис. 7. Трехмерная модель асинхронного электродвигателя, встроенного в маховик
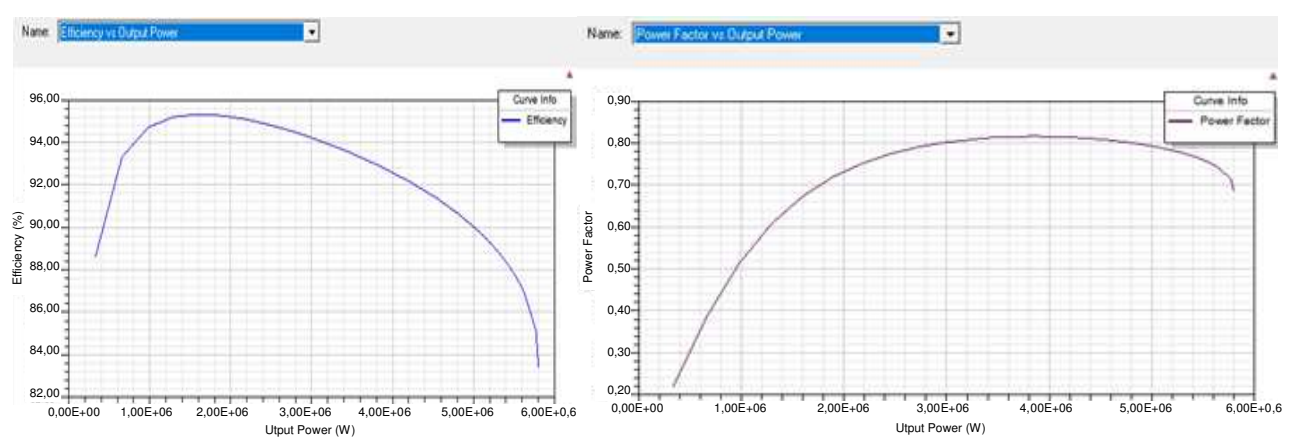

Рис. 8. Коэффициент мощности и КПД радиального асинхронного электродвигателя 2750 кВт, встраиваемого в маховик

Вариант имеет следующие основные достоинства:

- минимальные затраты на электродвигатель, так как для монтажа используются существующие детали;

- габариты позволяют разместить два независимых привода;

- вариант имеет небольшие технические риски, так как в случае невыполнения им своих функций остается работоспособным старый привод.

К недостаткам варианта следует отнести следующее:

- существующие замеренные биения заставляют делать большим воздушный зазор. Это снижает коэффициент мощности, что приводит к увеличению намагничивающего тока, повышению электрических потерь, снижению КПД;

- большая трудоемкость монтажа электродвигателя из сегментов на рабочем месте, сложность регулировки воздушного зазора. 
Торцевой асинхронный электродвигатель 2750 кВТ, 600 В, 35 об/мин, встраиваемый в существующий маховик. Большой диаметр маховика позволяет рассмотреть возможность установки в него торцевого двигателя. Особенностью торцевых двигателей является сильное одностороннее осевое тяжение, которое приводит к дополнительной нагрузке на подшипники [16-19]. Чтобы устранить этот фактор, было решено использовать два концевых двигателя, которые расположены по обе стороны от маховика и взаимно компенсируют это осевое тяжение.

Результаты расчета торцевых асинхронных электродвигателей приведены на рис. 9. Воздушный зазор был принят 10 мм, так как торцевые биения маховика составили 6 мм.
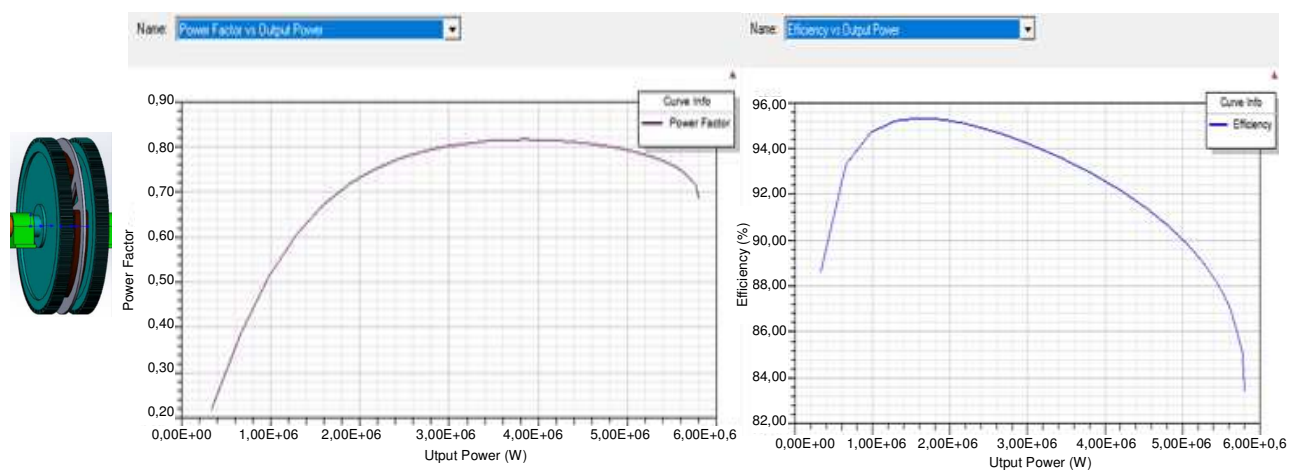

Рис. 9. Трехмерная модель, коэффициент мощности и КПД асинхронного электродвигателя 2750 кВт, встраиваемого в маховик

Конечный двигатель имеет аналогичные характеристики по сравнению с радиальным двигателем и имеет те же преимущества и недостатки.

\section{5. Исключение маховика и замена его электродвигателем} большого диаметра с эквивалентной инерционной массой. Преимущество этих электродвигателей в том, что они производятся и испытываются на специализированных предприятиях. В то же время технические риски изготовления электродвигателя с неудовлетворительными параметрами сводятся к минимуму. Кроме того, исключение маховика как отдельного конструктивного элемента экономит место для установки и, как следствие, вмещает два независимых привода.

Асинхронный электродвигатель 1375 кВт с эквивалентной инерционной массой. Результаты анализа данного варианта представлены на рис. 10 и 11. 


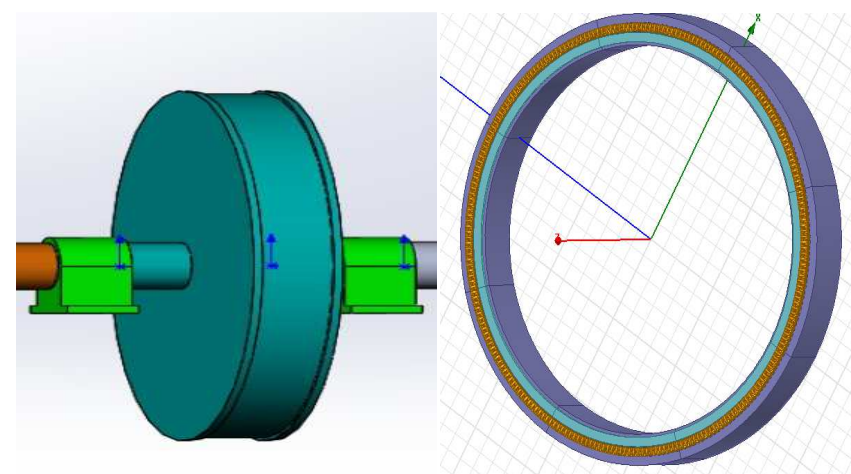

Рис. 10. Трехмерная модель асинхронного электродвигателя 1375 кВт и его активных частей
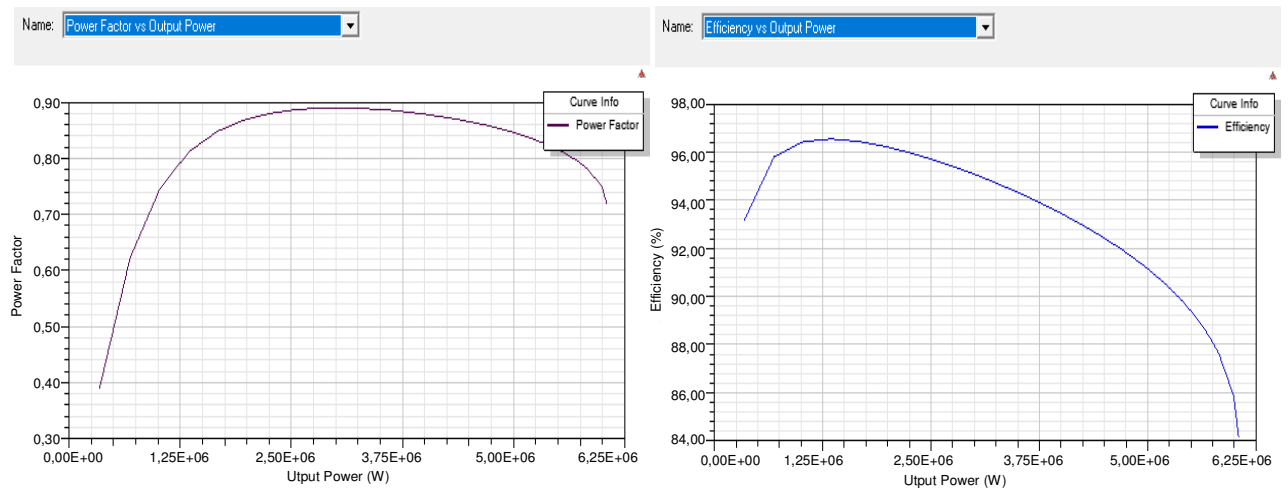

Рис. 11. Коэффициент мощности и КПД асинхронного электродвигателя с эквивалентной инерционной массой 1375 кВт

Достоинства варианта:

- электродвигатель изготавливается на специализированном предприятии с гарантией, поставляется как готовое комплектующее изделие;

- габариты позволяют разместить второй независимый привод;

- электродвигатель имеет закрытое исполнение, что уменьшает шум, повышает безопасность и надежность;

- асинхронные электродвигатели на данную мощность могут изготовить в Челябинске на предприятии АО «Русские электрические электродвигатели» (АО РЭД), которое имеет для этого все необходимое технологическое оборудование.

Недостатком этого варианта является низкий коэффициент мощности, что приводит к увеличению тока намагничивания, увеличению потерь и снижению КПД. 


\section{Вентильный электродвигатель с постоянными магнитами} с когтеобразными полюсами 1375 кВТ с эквивалентной инерционной массой. Электродвигатели с постоянными магнитами используются в качестве приводов на промышленных предприятиях. Эти электродвигатели имеют высокую эффективность, высокую перегрузочную способность, большие весовые и габаритные параметры, высокий коэффициент мощности (более 0,95) [9]. Также эти электродвигатели имеют жесткие механические характеристики.

Существенным недостатком машин с когтеобразными полюсами является сложность сборки статора и ротора из-за наличия мощных высококоэрцитивных постоянных магнитов. Одностороннее магнитное тяжение для крупногабаритных электродвигателей может составлять несколько тонн, что делает технологически невозможным монтаж.

Следует отметить, что конструкция машин с когтеобразными полюсами может решить эту техническую проблему. Для этого необходимо организовать сборку по следующей технологической цепочке:

- первая часть магнитопровода когтеобразных полюсов размещается в двигателе в своих подшипниках. Эта часть находится внутри статора без постоянных магнитов. В этом случае одностороннее натяжение ротора к статору отсутствует;

- далее постоянные магниты приклеиваются к этой части магнитопровода. При этом появляется магнитное тяжение ротора к статору, но оно компенсируется подшипниками;

- на заключительном этапе сборки встраивается вторая часть магнитопровода когтеобразных полюсов. Под действием магнитных сил она втягивается в ротор, завершая сборку.

Электродвигателю не требуется тратить дополнительную энергию для создания возбуждения, так как эту функцию выполняют постоянные магниты, поэтому он имеет наивысший из рассмотренных вариантов КПД.

На рис. 12-13 представлена трехмерная модель электродвигателя и его активных частей, а также его основные характеристики.

Вариант имеет следующие достоинства:

- минимальные потери и наибольший КПД;

- электродвигатель изготавливается на специализированном предприятии с гарантией, поставляется как готовое комплектующее изделие; 

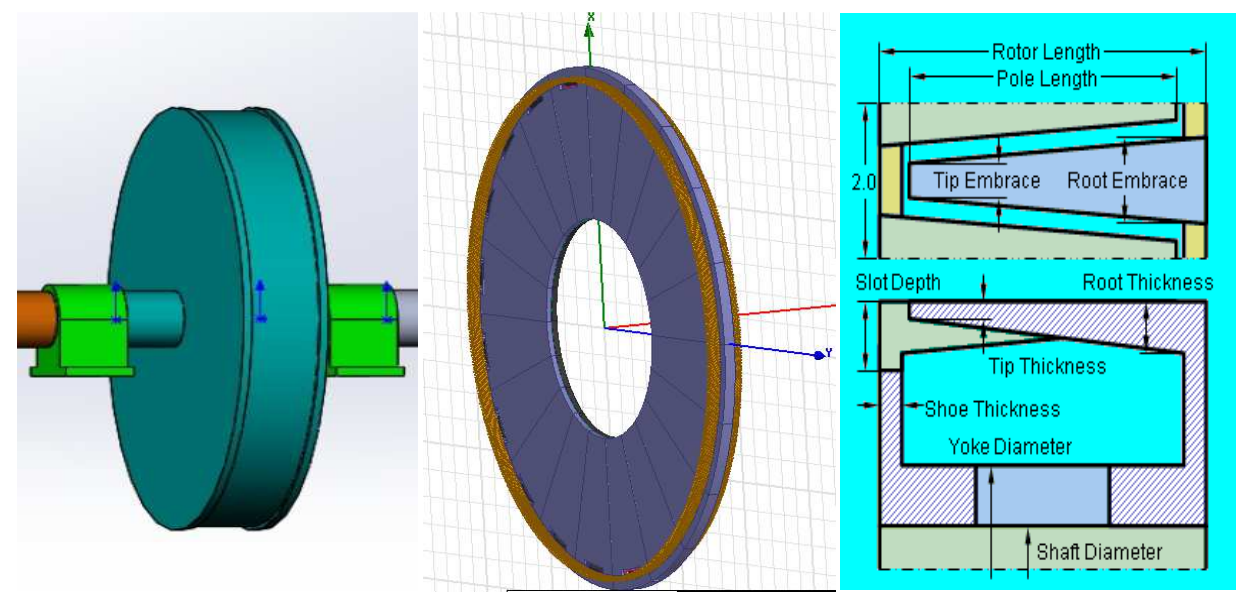

Рис. 12. Трехмерная модель и геометрия синхронного электродвигателя с когтеобразными полюсами 1375 кВт с эквивалентной инерционной массой

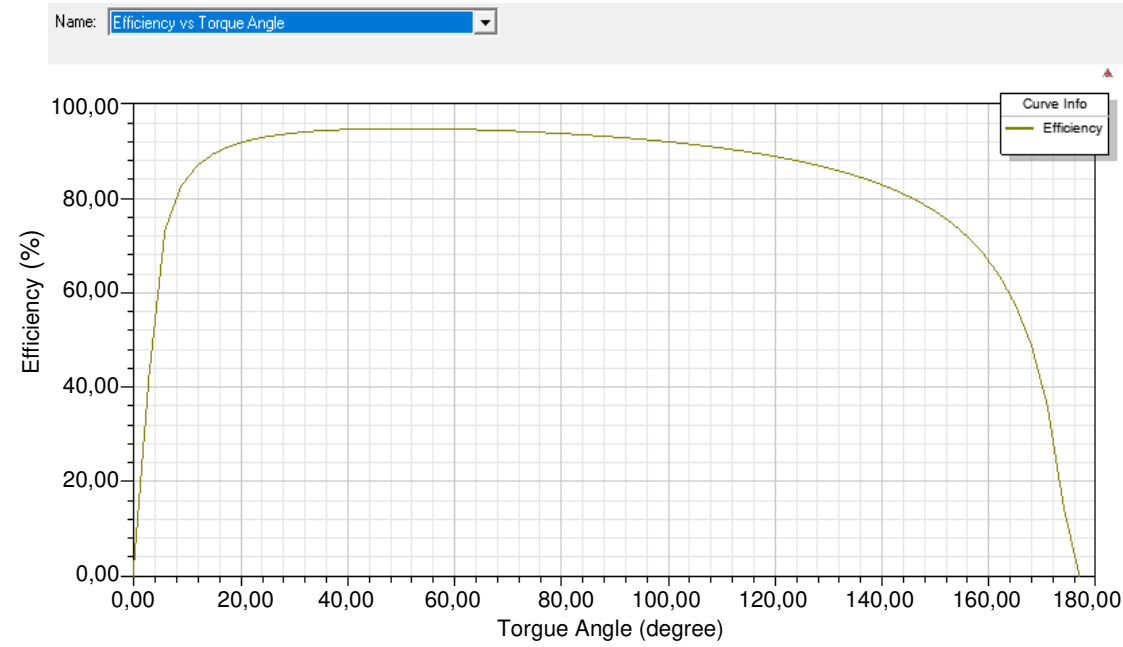

Рис. 13. КПД вентильного электродвигателя с когтеобразными полюсами 1375 кВт с эквивалентной инерционной массой

- конструкция позволяет разместить постоянные магниты без изготовления дополнительной оснастки;

- габариты одного привода с этим электродвигателем позволяют разместить второй независимый привод;

- электродвигатель имеет закрытое исполнение, что уменьшает шум, повышает безопасность и надежность;

- возможно изготовление электродвигателя на данную мощность в Челябинске на АО РЭД. 
К недостаткам варианта следует отнести следующее:

- электродвигатель содержит мощные дорогостоящие магниты;

- электродвигатель имеет реактивный момент, который будет создавать дополнительные вибрации на подшипники

\section{6. Сравнительный анализ вариантов. Выбор основного базо-} вого варианта. В качестве показателей наилучшего варианта примем минимальный расход активных материалов (медь) и энергетические показатели (КПД). Расход железа в качестве показателя экономии активных материалов не рассматривался, поскольку варианты были спроектированы, исходя из необходимого момента инерции с добавлением требуемой массы. Для вариантов электродвигателя мощностью 1375 кВт по расходу материалов было учтено, что для полной замены потребуются два привода и, соответственно, два электродвигателя.

Результаты анализа сведены в таблице.

Результаты анализа вариантов

\begin{tabular}{|c|c|c|c|}
\hline $\begin{array}{c}\text { № } \\
\text { п/п }\end{array}$ & Наименование варианта & $\begin{array}{c}\text { Масса } \\
\text { меди, кг }\end{array}$ & КПд \\
\hline 1 & Замена существующего электродвигателя постоянного тока на асин- \\
хронный электродвигатель 2750 кВт & 11570 & 0,955 \\
\hline 2 & $\begin{array}{c}\text { Замена существующего электродвигателя постоянного тока на синхрон- } \\
\text { ный электродвигатель 2750 кВт с электромагнитным возбуждением }\end{array}$ & 6600 & 0,945 \\
\hline 3 & Встраивание нового асинхронного радиального электродвигателя \\
2750 кВт в маховик & 11860 & 0,945 \\
\hline 4 & $\begin{array}{c}\text { Исключение маховика и замена его двумя асинхронными электродвигате- } \\
\text { лями 1375 кВт большого диаметра с эквивалентной инерционной массой }\end{array}$ & 11870 & 0,955 \\
\hline $\mathbf{5}$ & $\begin{array}{c}\text { Исключение маховика и замена его двумя вентильными электро- } \\
\text { двигателями с когтеобразными полюсами 1375 кВт большого } \\
\text { диаметра с эквивалентной инерционной массой }\end{array}$ & $\mathbf{5 6 0 0}$ & $\mathbf{0 , 9 7}$ \\
\hline
\end{tabular}

Из представленной таблицы видно, что вариант электродвигателя с когтеобразными полюсами является наилучшим с точки зрения расхода активных материалов и с точки зрения КПД. Это можно объяснить тем, что этот электродвигатель не расходует энергию на создание возбуждения за счет использования мощных постоянных магнитов. Исключение обмотки возбуждения также приводит к экономии меди.

Таким образом, на основе анализа рассмотренных вариантов в качестве базового для дальнейших уточненных электромагнитных и тепловых расчетов рекомендуется вариант двигателя с когтеобразными полюсами с постоянными магнитами. Он обладает наилучшими характеристиками при минимальном потреблении дорогостоящей меди и максимальном значении КПД, а также позволяет выполнить требования заказчика по изготовлению двух независимых приводов. 
7. Научная новизна выбранного базового варианта. Конструкция электрической машины с полюсами когтя известна и описана в многочисленных работах [7-9]. Эта электрическая машина в основном используется в качестве генератора с контактной системой возбуждения в виде кольцевой обмотки мощностью до 10 кВт. Использование данной конструкции в режиме электродвигателя с постоянными магнитами мощностью более 1 МВт с наружным диаметром 9 м является уникальным в мировой практике.

Для электродвигателя предложена новая технология изготовления, для которой постоянные магниты вклеиваются в ротор на этапе сборки всего электродвигателя.

Исключение из привода маховика как отдельного элемента и замена его на инерционную массу самого электродвигателя - это новое техническое решение в этом типе привода, что потребует разработки подшипникового узла с системой охлаждения и смазки.

Изучение этих вопросов является научной новизной в разработке уникального в мировой металлургической практике привода по производству бесшовных труб. Детальное изучение конструкции является основой для дальнейших исследований.

\section{8. Практическая значимость предлагаемой концепции приво-}

да. Основным практическим результатом предлагаемой конструкции привода является возможность разделения существующего агрегата на два независимых привода, работающих на свой цех. Это позволит проводить ремонтные и профилактические работы без полной остановки производства труб.

Отсутствие контактного токоподвода с щеточно-коллекторным узлом значительно повышает надежность при одновременном снижении затрат на техническое обслуживание и ремонт.

Данный привод потребует использования меньшего количества дорогостоящего цветного металла (обмоточной меди).

Уменьшенные электрические потери позволят снизить затраты на эксплуатацию привода.

9. Оценка показателей экономической эффективности базового варианта. Оценим экономию энергии базового варианта за счет снижения потерь и, следовательно, повышения КПД. Ближайший вариант по сравнению с выбранным имеет КПД 0,955. Для базового варианта КПД составляет 0,97 . 
Соответственно экономия на потерях при выборе базового варианта составит:

$$
\Delta P=\frac{\mathrm{P}_{\text {дв.ном }}}{0,955}-\frac{\mathrm{P}_{\text {дв.ном }}}{0,97}=\frac{2750}{0,955}-\frac{2750}{0,97}=44,5 \kappa \mathrm{КТ} .
$$

При постоянной работе 330 дней в году с перерывом на техническое обслуживание и средней стоимостью электроэнергии 3 руб./кВт.ч годовая экономия составит:

$$
\exists_{\text {годовая }}=\Delta P \cdot 24 \cdot 330 \cdot 3=44.5 \cdot 24 \cdot 330 \cdot 3=1057320 \text { руб, }
$$

т.е. более 1 млн рублей в год по действующим тарифам.

Следует отметить, что базовый вариант рассчитан на питание от источника постоянного тока 6 кВ, работающего в цехе. В этом случае коммутатор для электродвигателя будет значительно дешевле, чем преобразователь частоты для асинхронного привода, благодаря устранению уже существующего звена постоянного тока. Этот фактор приводит к еще большей экономии для реализации базового варианта.

В целом привод должен окупиться, примерно, за 3-5 лет за счет выпуска высокорентабельной продукции - труб большого диаметра для нефтегазовой отрасли.

Выводы. Из большого числа возможных вариантов для существующего привода пильгерстана ЧТПЗ в качестве основного предлагается вариант электродвигателя большого диаметра с когтеобразными полюсами и постоянными магнитами с эквивалентной инерционной массой. Электродвигатель имеет номинальную мощность 1375 кВт и скорость вращения 35 об/мин. Планируется управлять электродвигателем от собственного коммутатора, который питается от источника постоянного тока, имеющегося в цехе. Габаритные размеры позволяют провести монтаж второго независимого привода. Использование этого варианта электродвигателя позволяет снизить эксплуатационные расходы за счет повышения КПД, повышения надежности за счет устранения контактного токоподвода, снижения затрат на техническое обслуживание за счет разделения существующего привода на два независимых привода. Предлагаемый вариант удовлетворяет основным требованиям заказчика в лице АО ЧТПЗ, который он предъявляет к новому приводу пильгерстана, предназначенному для замены существующего физически и морально устаревшего агрегата. 


\section{Библиографический список}

1. Григорьев М.А. Вентильный электропривод с синхронной реактивной машиной независимого возбуждения: монография. - Челябинск: Изд. центр ЮУрГУ, 2010. - 159 с.

2. Козаченко В.Ф., Лашкевич М.М. Вентильно-индукторный электропривод с независимым возбуждением для тягового применения // Электротехнические и компьютерные системы. - 2011. - № 3. C. $138-139$.

3. Никифоров Б.В., Пахомин С.А., Птах Г.К. Вентильноиндукторные электродвигатели для тяговых электроприводов // Электричество. - 2007. - № 2. - С. 34-38.

4. Туан Н.М., Хай Н.Ч. Основные достоинства реактивновентильных электродвигателей по сравнению с традиционными электродвигателями // Известия Тул. гос. ун-та. Технические науки. - 2014. № 8. - С. 184-187.

5. Козаченко В.Ф. Корпусов Д.Е. Электропривод на базе вентильных индукторных машин с электромагнитным возбуждением // Электронные компоненты. - 2005. - № 6. - С. 60-64.

6. Голландцев, Ю.А. Сравнение механических характеристик асинхронных и вентильных индукторно-реактивных электродвигателей // Информационно-управляющие системы. - 2006. - № 6. - С. 50-53.

7. Копылов И.П. Электрические машины: учебник. - 5-е изд. М.: Высшая школа, 2006. - 607 с.

8. Вольдек А.И. Электрические машины: учебник. - 3-е изд. - Л.: Энергия, 1978. - 832 с.

9. Костенко М.П., Пиотровский Л.М. Электрические машины. Ч. 1: Машины постоянного тока. Трансформаторы. - Л.: Энергия, 1972. 544 с. Ч. 2: Машины переменного тока. - Л.: Энергия, 1973. - 648 с.

10. High-speed electrical machines: Technologies trends and developments / D. Gerada, A. Mebarki, N. Brown, C. Gerada, A. Cavagnino, A. Boglietti // IEEE Trans. Ind. Electron. - Jun. 2014. - Vol. 61. - № 6. P. 2946-2959.

11. A high-speed permanent-magnet machine for fault-tolerant drivetrains / L. Papini, T. Raminosoa, D. Gerada, C. Gerada // IEEE Trans. Ind. Electron. - Jun. 2014. - Vol. 61. - №. 6. - P. 3071-3080.

12. Сравнительный обзор синхронного электродвигателя с постоянными магнитами и бесколлекторного электродвигателя постоян- 
ного тока при непосредственном управлении моментом / И.В. Гуляев, А.В. Волков, А.А. Попов, Е.И. Ионова [и др.] // Научно-технический вестник Поволжья. - 2015. - № 6. - С. 123-128.

13. Опейко О.Ф., Пташкин А.И., Хильмон В.И. Тяговый электропривод с бездатчиковой системой векторного управления // Энергетика. Известия высш. учеб. завед. и энергетич. объединений СНГ. 2010. - № 6. - С. 37-43.

14. Гуляев И.В., Тутаев Г.М. Системы векторного управления электроприводом на основе асинхронизированного вентильного электродвигателя: монография. - Саранск: Изд-во Мордов. ун-та, 2010. - 200 с.

15. Kovalev K., Ivanov N., Tulinova E. Magnetic field distribution in the active zone of synchronous generators with electromagnetic excitation // International Conference on Industrial Engineering, Applications and Manufacturing. ICIEAM 2017. - Proceedings 8076353.

16. Gandzha S.A., Halstead R.L. Optimal design of brushless axial gap electric machines for low power windmills // Design World (engineering solution for product manufactures). - 2012. - № 1. - URL: www.designworldonline.com

17. Gandzha S.A., Kiessh I.E.Application brushless machines with combine excitation for a small and medium power windmills // Procedia Engineering. - December 2015. - 129:191-194.

18. Gandzha S.A., Kiessh I.E. Varible speed power. Procedia Engineering. - December 2015. - 129:731-735.

19. Gandzha S.A., Sogrin A.I., Kiessh I.E. The Comparative Analysis of Permanent Magnet Electric Machines with Integer and Fractional Number of Slots per Pole and Phase // Procedia Engineering. - December 2015. - 129:408-414.

20. Мартьянов А.С., Неустроев Н.И. Анализ электромеханических систем с помощью ANSYS Maxwell // Альтернативная энергетика и экология: междунар. науч. журнал. - 2014. - № 19(159). C. 47-52.

21. Babu B.M., Srinivas L.R., Bindhu B. A MLI topology with different braking mechanisms employing BLDC drive // IEEE International Conference on Power, Control, Signals and Instrumentation Engineering, ICPCSI. Chennai, India, 2017. - P. 1845-1849. DOI:10.1109/ICPCSI.2017.8392034

22. Speed control of high-performance brushless DC motor drives by load torque estimation / Ki-Hong Park, Tae-Sung Kim, Sung-Chan Ahn, 
Dong-Seok Hyun // IEEE 34th Annual Conference on Power Electronics Specialist. PESC '03. - Acapulco, Mexico. - 2003. - № 4. - P. 1677-1681. DOI: 10.1109/PESC.2003.1217709

23. Application of digital twins technology for the analysis of brushless electric machines with axial magnetic flux / S.A. Gandzha, D.S. Aminov, I.E. Kiessh, B.I. Kosimov // Digital industry: state and prospects of development 2018: International scientific conference. DOI: 10.1109/GloSIC.2018.8570132

24. Gandzha S.A., Aminov D.S., Kosimov B.I. Design of Brushless Electric Machine with Axial Magnetic Flux Based on the Use of Nomograms // International Ural Conference on Green Energy (UralCon). 2018. - P. 282-287. DOI: 10.1109/URALCON.2018.8544320

\section{References}

1. Grigor'ev M.A. Ventil'nyi elektroprivod s sinkhronnoi reaktivnoi mashinoi nezavisimogo vozbuzhdeniia [Brushless Direct Current Motor with synchronous reactive machine of independent excitation]. Cheliabinsk: Iuzhno-Ural'skii gosudarstvennyi universitet, 2010. 159 p.

2. Kozachenko V.F., Lashkevich M.M. Ventil'no-induktornyi elektroprivod s nezavisimym vozbuzhdeniem dlia tiagovogo primeneniia [Switched reluctance drive with external excitation for the traction electric drive]. Elektrotekhnicheskie i komp'iuternye sistemy, 2011, no. 3, pp. 138-139.

3. Nikiforov B.V., Pakhomin S.A., Ptakh G.K. Ventil'no-induktornye elektrodvigateli dlia tiagovykh elektroprivodov [Switched reluctance motors for traction electric drives]. Elektrichestvo, 2007, no. 2, pp. 34-38.

4. Tuan N.M., Khai N.Ch. Osnovnye dostoinstva reaktivnoventil'nykh elektrodvigatelei po sravneniiu $\mathrm{s}$ traditsionnymi elektrodvigateliami [The main advantages of switched reluctance motors compared to traditional motors]. Izvestiia Tul'skogo gosudarstvennogo universiteta. Tekhnicheskie nauki, 2014, no. 8, pp. 184-187.

5. Kozachenko V.F. Korpusov D.E. Elektroprivod na baze ventil'nykh induktornykh mashin s elektromagnitnym vozbuzhdeniem [Electric drive on the basis of switched reluctance motors with electromagnetic excitation]. Elektronnye komponenty, 2005, no. 6, pp. 60-64.

6. Gollandtsev, Iu.A. Sravnenie mekhanicheskikh kharakteristik asinkhronnykh i ventil'nykh induktorno-reaktivnykh elektrodvigatelei [Comparison of mechanical characteristics of asynchronous and switched reluc- 
tance inductor-reactive electric motors]. Informatsionno-upravliaiushchie sistemy, 2006, no. 6, pp. 50-53.

7. Kopylov I.P. Elektricheskie mashiny [Electrical machines]. 5nd ed. Moscow: Vysshaia shkola, 2006. 607 p.

8. Vol'dek A.I. Elektricheskie mashiny [Electrical machines]. 3nd ed. Leningrad: Energiia, 1978. 832 p.

9. Kostenko M.P., Piotrovskii L.M. Elektricheskie mashiny. Part 1. Mashiny postoiannogo toka. Transformatory [Electrical machines. Part 1. DC machines. Transformers]. Leningrad: Energiia, 1972, 544 p. Part 2. Mashiny peremennogo toka [Part 2. AC machines]. Leningrad: Energiia, 1973. 648 p.

10. Gerada D., Mebarki A., Brown N., Gerada C., Cavagnino A., Boglietti A. High-speed electrical machines: Technologies trends and developments. IEEE Trans. Ind. Electron. Jun. 2014, vol. 61, no. 6, pp. 2946-2959.

11. Papini L., Raminosoa T., Gerada D., Gerada C. A high-speed permanent-magnet machine for fault-tolerant drivetrains. IEEE Trans. Ind. Electron. Jun. 2014, vol. 61, no. 6, pp. 3071-3080.

12. Guliaev I.V., Volkov A.V., Popov A.A., Ionova E.I. et al. Sravnitel'nyi obzor sinkhronnogo elektrodvigatelia s postoiannymi magnitami i beskollektornogo elektrodvigatelia postoiannogo toka pri neposredstvennom upravlenii momentom [Comparative review of synchronous motor with permanent magnets and brushless DC motor with direct torque control]. Nauchno-tekhnicheskii vestnik Povolzh'ia, 2015, no. 6, pp. 123-128.

13. Opeiko O.F., Ptashkin A.I., Khil'mon V.I. Tiagovyi elektroprivod $\mathrm{s}$ bezdatchikovoi sistemoi vektornogo upravleniia [Traction motor with sensorless vector control system]. Energetika. Izvestiia vysshikh uchebnykh zavedenii i energeticheskikh ob"edinenii SNG, 2010, no. 6, pp. 37-43.

14. Guliaev I.V., Tutaev G.M. Sistemy vektornogo upravleniia elektroprivodom na osnove asinkhronizirovannogo ventil'nogo elektrodvigatelia [Motor vector control systems based on asynchronized BLDC motor]. Saransk: Mordovskii universitet, 2010. 200 p.

15. Kovalev K., Ivanov N., Tulinova E. Magnetic field distribution in the active zone of synchronous generators with electromagnetic excitation. International Conference on Industrial Engineering, Applications and Manufacturing. ICIEAM 2017. Proceedings 8076353.

16. Gandzha S.A., Halstead R.L. Optimal design of brushless axial gap electric machines for low power windmills. Design World (engineering solution for product manufactures), 2012, no. 1, available at: www.designworldonline.com 
17. Gandzha S.A., Kiessh I.E. Application brushless machines with combine excitation for a small and medium power windmills. Procedia Engineering. December 2015. 129:191-194.

18. Gandzha S.A., Kiessh I.E. Varible speed power. Procedia Engineering. December 2015, 129:731-735.

19. Gandzha S.A., Sogrin A.I.,Kiessh I.E. The Comparative Analysis of Permanent Magnet Electric Machines with Integer and Fractional Number of Slots per Pole and Phase. Procedia Engineering. December 2015, 129:408-414.

20. Mart'ianov A.S., Neustroev N.I. Analiz elektromekhanicheskikh sistem s pomoshch'iu ANSYS MAXWELL [Analysis of Electromechanical systems using ANSYS Maxwell]. Al'ternativnaia energetika $i$ ekologiia. Mezhdunarodnyi nauchnyi zhurnal, 2014, no. 19(159), pp. 47-52.

21. Babu B.M., Srinivas L.R., Bindhu B. A MLI topology with different braking mechanisms employing BLDC drive. IEEE International Conference on Power, Control, Signals and Instrumentation Engineering, ICPCSI. Chennai, India, 2017, pp. 1845-1849. DOI:10.1109/ICPCSI.2017.8392034

22. Ki-Hong Park, Tae-Sung Kim, Sung-Chan Ahn, Dong-Seok Hyun. Speed control of high-performance brushless DC motor drives by load torque estimation. IEEE 34th Annual Conference on Power Electronics Specialist,. PESC '03. Acapulco, Mexico, 2003, no. 4, pp. 1677-1681. DOI: 10.1109/PESC.2003.1217709

23. Gandzha S.A., Aminov D.S., Kiessh I.E., Kosimov B.I. Application of digital twins technology for the analysis of brushless electric machines with axial magnetic flux. Digital industry: state and prospects of development 2018. International scientific conference 2018. DOI: 10.1109/GloSIC.2018.8570132

24. Gandzha S.A., Aminov D.S., Kosimov B.I. Design of Brushless Electric Machine with Axial Magnetic Flux Based on the Use of Nomograms. International Ural Conference on Green Energy (UralCon), 2018, pp. 282-287. DOI: 10.1109/URALCON.2018.8544320

\section{Сведения об авторах}

Ганджа Сергей Анатольевич (Челябинск, Россия) - доктор технических наук, заведующий кафедрой «Теоретические основы электротехники» Южно-Уральского государственного университета (454080, Челябинск, пр. Ленина, 76, e-mail: gandja_sa@mail.ru). 
Аминов Дилшод Саидович (Челябинск, Россия) - аспирант кафедры «Теоретические основы электротехники» Южно-Уральского государственного университета (454080, Челябинск, пр. Ленина, 76, e-mail: dilshod-aminov-93@mail.ru).

Косимов Бахтиёр Исматуллоевич - аспирант кафедры «Теоретические основы электротехники» Южно-Уральского государственного университета (454080, Челябинск, пр. Ленина, 76, e-mail: kosimov.energy@mail.ru).

Ниматов Рустам Рамазонович (Комсомольск-на-амуре, Россия) - аспирант кафедры «Промышленная электроника» Комсомольского-на-амуре государственного университета (681013, Комсомольск-на-Амуре, пр. Ленина, 27, e-mail: rustam0592@mail.ru).

\section{About the authors}

Gandzha Sergey Anatolyevich (Chelyabinsk, Russian Federation) is a Doctor of Technical Sciences, Head of the Department of Theoretical Fundamentals of Electrotechnology of South Ural State University (454080, Chelyabinsk, 76, Lenina ave., e-mail: gandja_sa@mail.ru).

Aminov Dilshod Saidovich (Chelyabinsk, Russian Federation) is a Graduate Student of Department of Theoretical Fundamentals of Electrotechnology of South Ural State University (454080, Chelyabinsk, 76, Lenina ave., e-mail: dilshod-aminov-93@mail.ru).

Kasimov Bakhtiyor Ismatulloevich (Chelyabinsk, Russian Federation) is a Graduate Student of Department of Theoretical Fundamentals of Electrotechnology of South Ural State University (454080, Chelyabinsk, 76, Lenina ave., e-mail: kosimov.energy@mail.ru).

Nimatov Rustam Ramazanovich (Komsomolsk-on-Amur, Russian Federation) is a Graduate Student of the Department of Industrial electronics of Komsomolsk-on-Amur State University (681013, Komsomolsk-onAmur, 27, Lenina ave., e-mail: rustam0592@mail.ru).

Получено 15.04.2019 